\title{
Comparison of Partec Rapid Malaria Test with Conventional Light Microscopy for Diagnosis of Malaria in Northwest Ethiopia
}

\author{
Meseret Birhanie \\ Department of Medical Parasitology, School of Biomedical and Laboratory Sciences, College of Medicine and Health Sciences, \\ University of Gondar, P.O. Box 196, Gondar, Ethiopia
}

Correspondence should be addressed to Meseret Birhanie; mesibir21@gmail.com

Received 30 September 2015; Revised 10 December 2015; Accepted 17 December 2015

Academic Editor: D. S. Lindsay

Copyright (C) 2016 Meseret Birhanie. This is an open access article distributed under the Creative Commons Attribution License, which permits unrestricted use, distribution, and reproduction in any medium, provided the original work is properly cited.

\begin{abstract}
Background. Laboratory diagnosis of malaria is the key for effective disease management. Diagnosis of malaria infection requires rapid, sensitive, and specific test methods with an affordable cost. This study was aimed to assess the diagnostic performance of Partec rapid malaria test with reference to light microscopy for the diagnosis of malaria in Northwest Ethiopia. Methods. A total of 180 febrile patients were tested for malaria using Giemsa stain microscopy and Partec rapid malaria test from June to July 2013 at Gendewuha health centers, Metema district. Data were analyzed using SPSS version 20 statistical software. Odds ratio with $95 \%$ CI was calculated. Result. The sensitivity and specificity of Partec rapid malaria test were $93.8 \%(95 \% \mathrm{CI}=87.1 \%-100 \%)$ and $87.9 \%(95 \%$ $\mathrm{CI}=79.7 \%-96.1 \%)$, respectively, while the positive predictive value and negative predictive value were $6.4 \%(95 \% \mathrm{CI}=77.2 \%-95.5 \%)$ and $94.6 \%(95 \% \mathrm{CI}=88.7 \%-100 \%)$, respectively. There was also an excellent agreement between two tests with Kappa value of 0.811 (95\% CI = 0.625-0.996). Conclusion. Partec rapid malaria test showed good sensitivity and specificity with an excellent agreement to the reference light microscopy. Therefore PT can be considered as alternative diagnostic tools in malaria endemic areas.
\end{abstract}

\section{Background}

Malaria is the most common fatal disease in the world. According to 2013 WHO malaria report, an estimated 3.4 billion people were at risk of malaria globally and populations living in sub-Saharan Africa have the highest risk of acquiring malaria. Approximately $80 \%$ of cases and $90 \%$ of deaths are estimated to occur in the African region [1]. In Ethiopia approximately 52 million people (68\%) live in malaria risk areas. Plasmodium falciparum and $P$. vivax are the dominant species of malaria in Ethiopia, with $60 \%$ and $40 \%$ relative frequencies, respectively. $P$. falciparum is the most dominant species in endemic areas and causes severe and complicated disease and death [2].

In Ethiopia half a million microscopically confirmed cases of malaria are reported to the Federal Ministry of Health (FMOH) in each year from basic health services. However, the actual number of malaria cases in the country is estimated to be more than 5 million each year [2]. So, choosing an accurate, rapid, and cost-effective diagnostic test is mainstay of efficient clinical and epidemiological management of malaria $[3,4]$.

Clinical diagnosis is the most widely used diagnostic method in rural areas and where laboratory facility does not exist. It is inexpensive to perform and requires no special equipment or supplies [2]. However, according to WHO 2011 guidelines clinical diagnosis of malaria based on signs and symptoms alone is not recommended since it has low specificity and increases the chances of the patient being misdiagnosed and leads to misuse of drugs $[5,6]$.

A laboratory diagnosis of malaria is one possibility in the management of a patient presenting with fever. To improve the quality care of the patients, many diagnostic procedures have been developed which aim to have accurate diagnosis, to reduce the time of preparation and training needed [3, 4]. Even though Giemsa stain microscopy remains a gold standard method for malaria diagnosis in many developing countries $[4,7,8]$, but it is not $100 \%$ sensitive and specific [9]. 
The Partec rapid malaria test (PT) is newly innovative fluorescence microscopy method for the detection of Plasmodium species DNA in human blood. It uses readily prepared and ready-to-use slides labeled with an unspecific DNA-binding fluorescent dye ( $4^{\prime}$-6-diamidino-2phenylindole (DAPI); emission $365 \mathrm{~nm}$ ) that detects plasmodial DNA. It is ultra compact and robust microscope design and has connector for optionally available CCD camera upgrade for visualization of the slides on any PC Windows with USB connection $[10,11]$. The sensitivity and specificity of the test were not assessed in Ethiopia. Thus this study was conducted to evaluate the performance of CyScope florescence microscopy (Partec rapid malaria test) in reference to light microscopy in Northwest Ethiopia.

\section{Methods}

2.1. Study Area and Period. A study was conducted at Gendewuha health center found in Metema district, North Gondar Zone, Amhara Regional State, from June to July 2013. The district is found at the Ethio-Sudan border. It is far $925 \mathrm{~km}$ from Addis Ababa (capital city of Ethiopia) and found at $180 \mathrm{~km}$ west of Gondar town. The mean annual temperature ranges from $22^{\circ} \mathrm{C}$ to $28^{\circ} \mathrm{C}$ and the daily temperature reaches as high as $43^{\circ} \mathrm{C}$ during the months of March to May. Mean annual rainfall ranges from $850 \mathrm{~mm}$ to $1100 \mathrm{~mm}$ and has unimodal pattern. The altitudes range from 550 to 1068 meters above sea level [12]. The district is malarious and endemic for all Plasmodium species. But the prevalence of $P$. falciparum is very high. The health center is serving around 18,000 people in Gendewuha town and surrounding areas.

2.2. Study Subject. One hundred and eighty malaria suspected patients attending at Gendewuha health center during the study period were included and screened for malaria infection using light microscopy and Partec rapid malaria test. Patients who had received antimalarial drugs during the past two weeks, children under 1 year, and critically ill patients who were unable to respond to the interview were excluded from the study.

2.3. Specimen Collection and Processing. Sociodemographic and clinical data of the study participants were collected using structured questionnaire. Three milliliters of venous blood was collected with EDTA tubes by laboratory technicians from each study subject for smear preparation of light microscopy and PT.

2.4. Light Microscopy. After collecting blood samples, thick and thin blood smears were prepared and stained with $10 \%$ Giemsa working solution for 10 minutes. Blood films were observed under 100x objectives for the detection of malaria parasites and the result was reported as positive if asexual malaria parasites were seen or negative if malaria parasites were not seen after observing 100 fields of the thick smear. All blood films were reexamined by an experienced microscopist at University of Gondar Hospital laboratory who was blinded to initial light microscopy result.
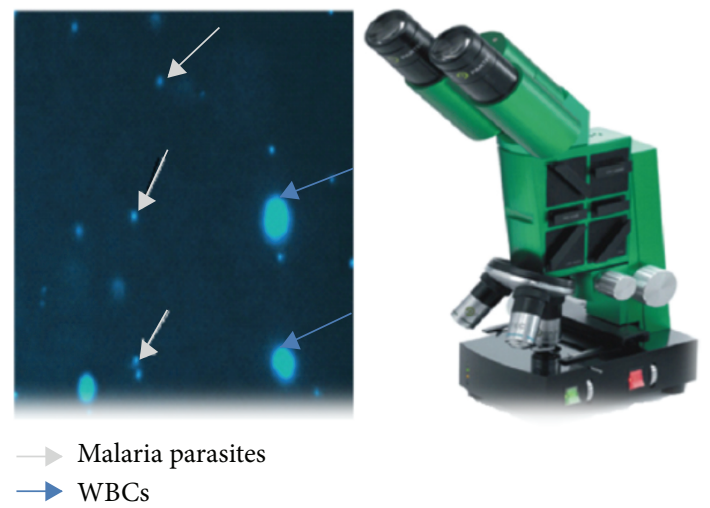

Figure 1: Partec CyScope fluorescence microscope and malaria parasites (adapted from [11]).

2.5. Partec Rapid Malaria Test. Partec rapid malaria test was carried out according to the manufacturer's instructions. Briefly, on the collected EDTA blood, $10 \mu \mathrm{L}$ blood was placed on the dye labeled area of a slide and cover slipped, incubated at room temperature for a minute, and observed with $40 \mathrm{x}$ objective under LED UV light $(365 \mathrm{~nm})$. The presence of bright shiny intracellular tiny dots against a dark background observed under the UV light indicated the presence of malaria parasites in the erythrocytes as shown in Figure 1.

2.6. Statistical Analysis. The data was entered into SPSS version 20 software for analysis. Odds ratio, sensitivity, specificity, positive predictive value, negative predictive value, and Kappa value were calculated at 95\% CI.

2.7. Ethical Approval. Ethical clearance was obtained from University of Gondar, School of Biomedical and Laboratory Sciences Ethical Committee, and permission was obtained from Gendewuha health office and Gendewuha health center authorities to conduct the study. After informing about the objective of the study, written consent was taken from all study participants or parents/guardians. Participants who were positive to malaria parasite were given the appropriate treatment at the health centre.

\section{Result}

A total of 180 malaria suspected patients were diagnosed for malaria. About 99 (55\%) of the study participants were males and $81(45 \%)$ were females. The mean age of the participants was $19 \pm 12.49$ years and majority of the participants 60 (33.3\%) were within the age range of 20-29 years, and $42.8 \%$ of the participants were illiterate. The rural residence has statistically significant association with malaria infection $(\mathrm{AOR}=0.437, \mathrm{CI}=0.028-0.835)($ Table 1$)$.

3.1. Light Microscopy. The overall prevalence of malaria using light microscopy was 81 (45\%). From this Plasmodium falciparum accounts for 76 (93.8\%), P. vivax were 4 (4.9\%), and mixed infection ( $P$. falciparum and $P$. vivax) was 1 $(1.3 \%)$. There were 2 discordant results between the first 
TABLE 1: Sociodemographic characteristics of patients and malaria positivity by light microscopy Gendewuha health center, Northwest Ethiopia, from June to July, 2013.

\begin{tabular}{|c|c|c|c|c|c|c|c|}
\hline \multirow{2}{*}{ Characteristics } & \multicolumn{7}{|c|}{ Light microscopy } \\
\hline & $\begin{array}{c}\text { Tested patients } \\
N(\%)\end{array}$ & $\begin{array}{c}\text { Positive } N \\
(\%)\end{array}$ & Negative $N(\%)$ & COR & $95 \% \mathrm{CI}$ & AOR & $95 \% \mathrm{CI}$ \\
\hline \multicolumn{8}{|l|}{ Gender } \\
\hline Male & $99(55.0)$ & $53(53.5)$ & $46(46.5)$ & 0.459 & $0.250-0.839$ & & \\
\hline Female & $81(45.0)$ & $28(34.6)$ & $53(65.4)$ & 1 & & & \\
\hline \multicolumn{8}{|l|}{ Age in years } \\
\hline $1-9$ & $51(28.3)$ & $15(29.4)$ & $36(70.6)$ & 1.029 & $0.234-4.521$ & & \\
\hline $10-19$ & $44(24.4)$ & $17(38.6)$ & $27(61.4)$ & 0.681 & $0.155-2.997$ & & \\
\hline $20-29$ & $60(33.3)$ & $42(70.0)$ & $18(30.0)$ & 0.199 & $0.046-0.853$ & 0.179 & $0.040-0.792$ \\
\hline $30-39$ & $15(8.3)$ & $5(33.3)$ & $10(66.7)$ & 0.857 & $0.152-4.819$ & & \\
\hline$\geq 40$ & $10(5.6)$ & $3(30.0)$ & $7(70.0)$ & 1 & & & \\
\hline \multicolumn{8}{|l|}{ Residence } \\
\hline Rural & $86(47.8)$ & $49(57.0)$ & $37(43.0)$ & 0.390 & $0.213-0.713$ & 0.437 & $0.0228-0.835$ \\
\hline Urban & $94(52.2)$ & $32(34.0)$ & $62(66.0)$ & 1 & & & \\
\hline \multicolumn{8}{|c|}{ Educational background } \\
\hline Illiterate & $76(42.2)$ & $39(51.3)$ & $37(48.7)$ & 0.632 & $0.100-4.00$ & & \\
\hline Read and write & $39(21.7)$ & $17(43.6)$ & $22(56.4)$ & 0.863 & $0.129-5.756$ & & \\
\hline Primary school & $51(28.3)$ & $21(41.2)$ & $30(58.8)$ & 1.033 & $0.158-6.741$ & & \\
\hline Secondary & $9(5.0)$ & $3(33.3)$ & $6(66.7)$ & 1.333 & $0.139-12.818$ & & \\
\hline College/university & $5(2.8)$ & $2(40.0)$ & $3(60.0)$ & 1 & & & \\
\hline \multicolumn{8}{|l|}{ Occupation } \\
\hline Farmer & $39(21.7)$ & $18(46.2)$ & $21(53.8)$ & 0.648 & $0.184-2.28$ & & \\
\hline Merchant & 34 (18.9) & $10(29.4)$ & $24(70.6)$ & 1.33 & $0.357-4.98$ & & \\
\hline Civil servant & $7(3.9)$ & $2(28.6)$ & $5(71.4)$ & 1.389 & $0.194-9.96$ & & \\
\hline Daily laborers & $47(26.1)$ & $31(66.0)$ & $16(34.0)$ & 0.287 & $0.082-0.99$ & & \\
\hline Housewife & $30(16.7)$ & $12(40.0)$ & $18(60.0)$ & 0.833 & $0.224-3.10$ & & \\
\hline Private & $9(5.0)$ & $4(44.4)$ & $5(55.6)$ & 0.694 & $0.126-3.83$ & & \\
\hline Student & $14(7.8)$ & $5(35.7)$ & $9(64.3)$ & 1 & & & \\
\hline
\end{tabular}

$\mathrm{N}=$ number $\mathrm{COR}=$ crude odds ratio; $\mathrm{AOR}=$ adjusted odds ratio; $\mathrm{CI}=$ confidence interval.

(study site) reader and the final reading by experienced microscopist; thus the final report was based on the final reading. Turnaround time of Giemsa stain microscopy was more than an hour.

3.2. Partec Rapid Malaria Test. The parasite positivity of malaria by using Partec rapid malaria test was 88 (48.9\%). Seventy-six slides were positive with Partec rapid malaria test and the gold standard Giemsa stain microscopy. Twelve slides were positive with Partec rapid malaria test and negative with light microscopy, while 5 slides were negative with Partec rapid malaria test and positive with light microscopy and 87 slides were negative for both (Table 2).

3.3. Sensitivity and Specificity. Using light microscopy as standard test for diagnosing malaria, the sensitivity and specificity of Partec rapid malaria test were 93.8\% (95\% CI = 87.1-100) and $87.9 \%$ (95\% CI $=79.7-96.1)$, respectively. There was also an excellent agreement between light microscopy
TABLE 2: Results of light microscopy and Partec rapid malaria test.

\begin{tabular}{lcccc}
\hline & & \multicolumn{3}{c}{ Light microscopy } \\
& & Positive & Negative & Total \\
\hline \multirow{2}{*}{ Partec rapid } & Positive & 76 & 12 & 88 \\
malaria test & Negative & 5 & 87 & 92 \\
& Total & 81 & 99 & 180 \\
\hline
\end{tabular}

and Partec rapid malaria test with Kappa value of 0.811 (95\% $\mathrm{CI}=0.625-0.996)($ Table 3$)$.

In addition, operational characteristics were observed. Compared to light microscopy PT is more sensitive, less labor intensive, and faster to use and has a less turnaround time than the LM (an average of 5 minutes). PT also requires very little training and there is no need of reagent preparation. Furthermore, the CyScope florescence microscope is battery operated; the battery can be kept on for 6 hours after full charge making it best for field work. It is easier to use and 
TABLE 3: Performance of Partec rapid malaria test using light microscopy as a standard.

\begin{tabular}{lccccc}
\hline Test & $\begin{array}{c}\text { Sensitivity\% } \\
(95 \% \mathrm{CI})\end{array}$ & $\begin{array}{c}\text { Specificity\% } \\
(95 \% \mathrm{CI})\end{array}$ & $\begin{array}{c}\text { PPV\% } \\
(95 \% \mathrm{CI})\end{array}$ & $\begin{array}{c}\text { NPV\% } \\
(95 \% \mathrm{CI})\end{array}$ & $\begin{array}{c}\text { Kappa value\% } \\
(95 \% \mathrm{CI})\end{array}$ \\
\hline Partec rapid & $93.8 \%$ & $87.9 \%$ & $86.4 \%$ & $94.6 \%$ & $81.1 \%$ \\
malaria test & $(87.1-100)$ & $(79.7-96.1)$ & $(77.2-95.5)$ & $(88.7-100)$ & $(62.5-99.6)$ \\
\hline
\end{tabular}

to adjust and can be switched from UV light to bright field. However, the PT is not suitable for species identification and parasitic load determination and blood films cannot be stored for a long period of time.

\section{Discussion}

The current study revealed a high sensitivity and specificity of Partec rapid malaria test. The high sensitivity and specificity are in agreement with the reports in Sudan [13]. However, the sensitivity and specificity are lower than the reports in Zimbabwe [14], Ghana [11, 15], Sudan [16], and Uganda [17]. These differences might be due to observer variation or host factors. Partec rapid malaria test had high PPV and NPV. Thus, a high NPV indicates that a person does not have the disease with high certainty, meaning that PT is reliable test method in diagnosing of malaria parasites.

Partec rapid malaria test had detected 12 cases which were negative by light microscopy. Unlike Giemsa stain light microscopy, PT has sensitive fluorescent dye, 4,6-diamidino2-phenylindole (DAPI), which can be detected in such low level of parasites. In addition, the presence of artifacts such as nonspecific aggregated DAPI, immature erythrocytes, or bacterial cells might have been misinterpreted as Plasmodium DNA [15]. On the other hand, PT produced 5 false negative results which were positive by light microscopy. This might be due to the fact that unlysed red blood cells may lie on each other or overlap with each other thereby preventing parasites in red blood cells that may be lying beneath other cells. The performance characteristics of the tests were very similar as indicated in their sensitivities, specificities, PPV, and NPV with very good agreements to the light microscopy.

Determination of parasitemia with Partec rapid malaria test is difficult, because the red blood cell may overlap each other and make the parasites invisible under the UV light. This finding is in line with the finding of the study conducted in Ghana [15]. Though, PT is useful as light microscopy thick film for screening purpose in malaria endemic areas.

The overall prevalence of malaria in the study area was very high, as detected by both the LM (45\%) and the PT $(48.9 \%)$. This result is higher than the report from other regions in Ethiopia [18, 19]. The high prevalence could be partly explained by the fact that the study was conducted in malaria transmission season of the country.

\section{Conclusion}

Partec rapid malaria test showed good sensitivity and specificity with an excellent agreement to the reference light microscopy. PT has very short turnaround times, requires little training, and is applicable under field conditions. Therefore PT can be considered as alternative diagnostic tools in malaria endemic areas. Further studies are needed to determine the cost-effectiveness and long term performance of the Partec rapid malaria test in diagnosing malaria.

\section{Abbreviations}

PT: $\quad$ Partec rapid malaria test

LM: Light microscopy

PPV: Positive predictive value

NPV: Negative predictive value

UV: Ultraviolet.

\section{Conflict of Interests}

The author declares that there is no conflict of interests.

\section{Authors' Contribution}

Meseret Birhanie supervised the data collection, performed laboratory tests, and analyzed and wrote the paper.

\section{Acknowledgments}

The author would like to extend their appreciation to Gendewuha health center laboratory professionals for their help in the collection of data and processing and analyzing of laboratory tests. Also the author wants to express their great thanks to the study participants for their patience and cooperation. Finally, the author would like to acknowledge the USAID ENHAT-CS program for the financial support of this research and Partec $\mathrm{GmbH}$, Germany, for providing the Partec CyScope microscope and the Partec rapid malaria test kits.

\section{References}

[1] WHO, World Malaria Report 2013, World Health Organization, Geneva, Switzerland, 2013.

[2] Federal Republic of Ethiopia Ministry of Health, National Malaria Guide Lines, FMOH, Addis Ababa, Ethiopia, 3rd edition, 2012.

[3] N. Tangpukdee, C. Duangdee, P. Wilairatana, and S. Krudsood, "Malaria diagnosis: a brief review," Korean Journal of Parasitology, vol. 47, no. 2, pp. 93-102, 2009.

[4] WHO, Universal Access to Malaria Diagnostic Testing. An Operational Manual, WHO, Geneva, Switzerland, 2011, http:// www.who.int/malaria/en/.

[5] WHO, World Malaria Report, 2011, http://www.who.int/malaria/ world_malaria_report_2011/WMR2011_factsheet.pdf. 
[6] T. Leslie, A. Mikhail, I. Mayan et al., "Over diagnosis and mistreatment of malaria among febrile patients at primary health care level in Afghanistan," British Medical Journal, vol. 345, Article ID e4389, 2012.

[7] A. Moody, "Rapid diagnostic tests for malaria parasites," Clinical Microbiology Reviews, vol. 15, no. 1, pp. 66-78, 2002.

[8] P. Lee and M. N. Shaio, "A review and comparison of malaria diagnostic tests," Kuang Tien Medical Journal, vol. 2, no. 4, pp. 57-64, 2007.

[9] B. S. C. Uzochukwu, L. O. Chiegboka, C. Enwereuzo et al., "Examining appropriate diagnosis and treatment of malaria: availability and use of rapid diagnostic tests and artemisininbased combination therapy in public and private health facilities in south east Nigeria," BMC Public Health, vol. 10, pp. 486-495, 2010.

[10] Partec Essential Healthcare, http://nsc-ksa.com/catalogue/ NSC\%20CATALOGUES/Flow\%20cytometers/Essential-Healthcare.pdf.

[11] B. Nkrumah, A. Agyekum, S. E. K. Acquah et al., "Comparison of the novel partec rapid malaria test to the conventional giemsa stain and the gold standard real-time PCR," Journal of Clinical Microbiology, vol. 48, no. 8, pp. 2925-2928, 2010.

[12] T. Desalew, A. Tegegne, L. Nigatu, and W. Teka, Rangeland Condition and Feedresources in Metema District, North Gondar Zone, Amhara Region, Ethiopia, International Livestock Research Institute, Nairobi, Kenya, 2010.

[13] S. E.-D. H. Hassan, A. E. D. Haggaz, E. B. Mohammed-Elhassan, E. M. Malik, and I. Adam, "Fluorescence microscope (Cyscope) for malaria diagnosis in pregnant women in Medani Hospital, Sudan," Diagnostic Pathology, vol. 6, no. 1, article 88, 2011.

[14] L. Gwanzura, J. Mayini, K. Mabhanga et al., "Evaluation of 'Cyscope, a novel fluorescence-based microscopy technique for the detection of malaria," Journal of Infections in Developing Countries, vol. 6, no. 2, pp. 212-215, 2011.

[15] N. Nkrumah, S. E. K. Acquah, L. Ibrahim et al., "Comparative evaluation of two rapid field tests for malaria diagnosis: Partec Rapid Malaria Test and Binax Now malaria rapid diagnostic test," BMC Infectious Diseases, vol. 11, article 143, 2011.

[16] S. El-Din Hassan, S. I. Okoued, M. A. Mudathir, and E. M. Malik, "Testing the sensitivity and specificity of the fluorescence microscope (Cyscope) for malaria diagnosis," Malaria Journal, vol. 9 , article 88, 2010.

[17] J. C. Sousa-Figueiredo, D. Oguttu, M. Adriko et al., "Investigating portable fluorescent microscopy (CyScope) as an alternative rapid diagnostic test for malaria in children and women of child-bearing age," Malaria Journal, vol. 9, no. 1, article 245, 2010.

[18] T. Endeshaw, T. Gebre, J. Ngondi et al., "Evaluation of light microscopy and rapid diagnostic test for the detection of malaria under operational field conditions: a household survey in Ethiopia," Malaria Journal, vol. 7, article 118, 2008.

[19] B. Moges, B. Amare, Y. Belyhun et al., "Comparison of CareStart HRP2/pLDH COMBO rapid malaria test with light microscopy in north-west Ethiopia," Malaria Journal, vol. 11, article 234, 2012. 

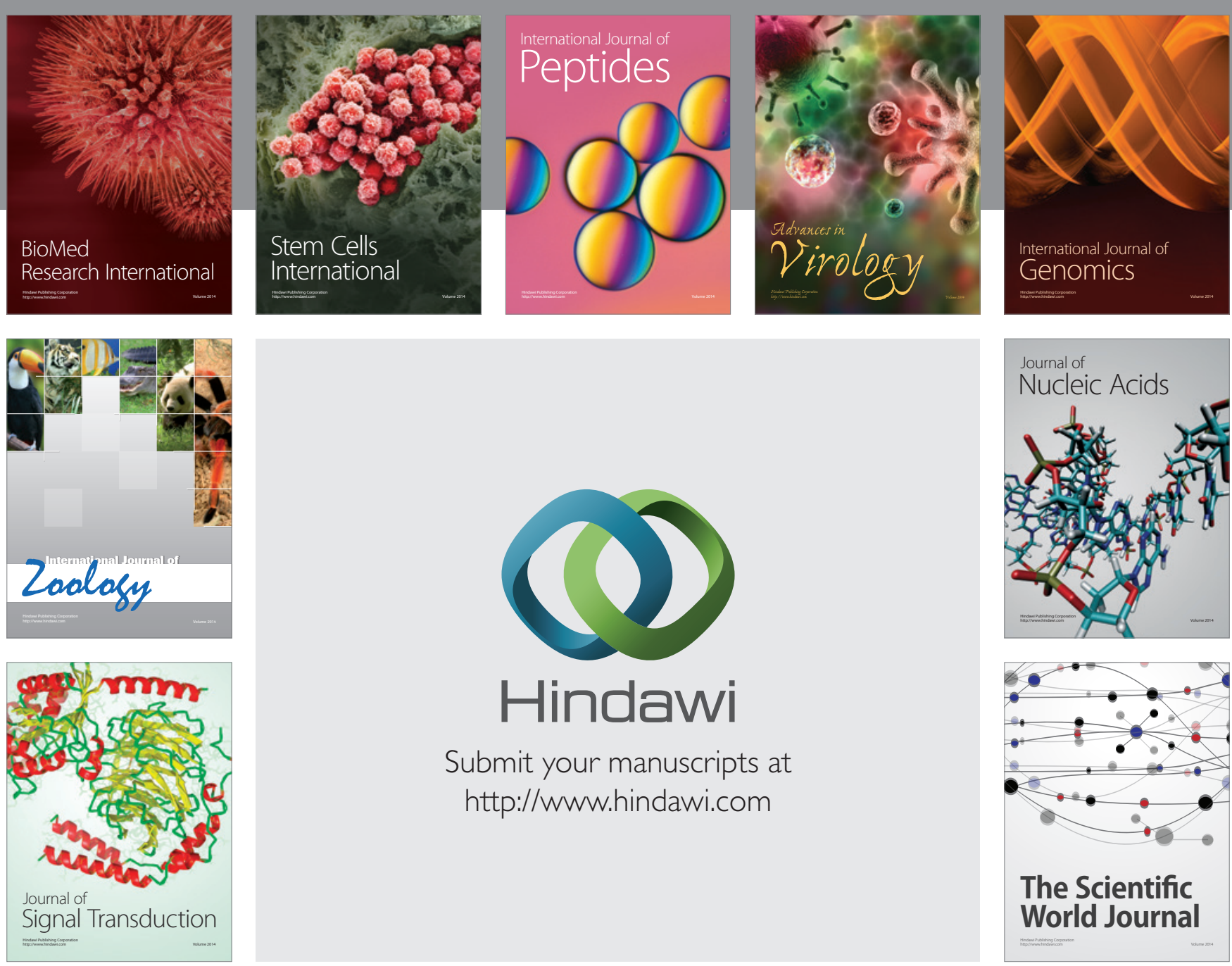

Submit your manuscripts at

http://www.hindawi.com
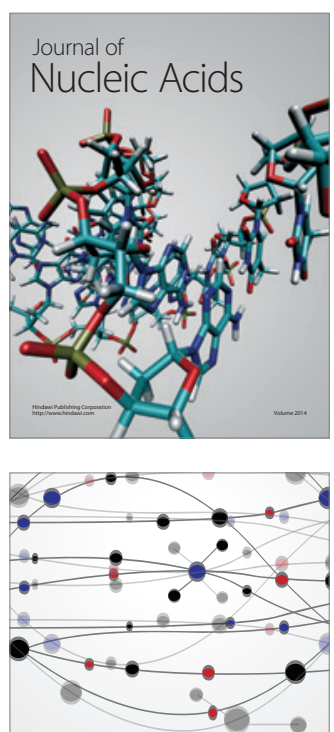

The Scientific World Journal
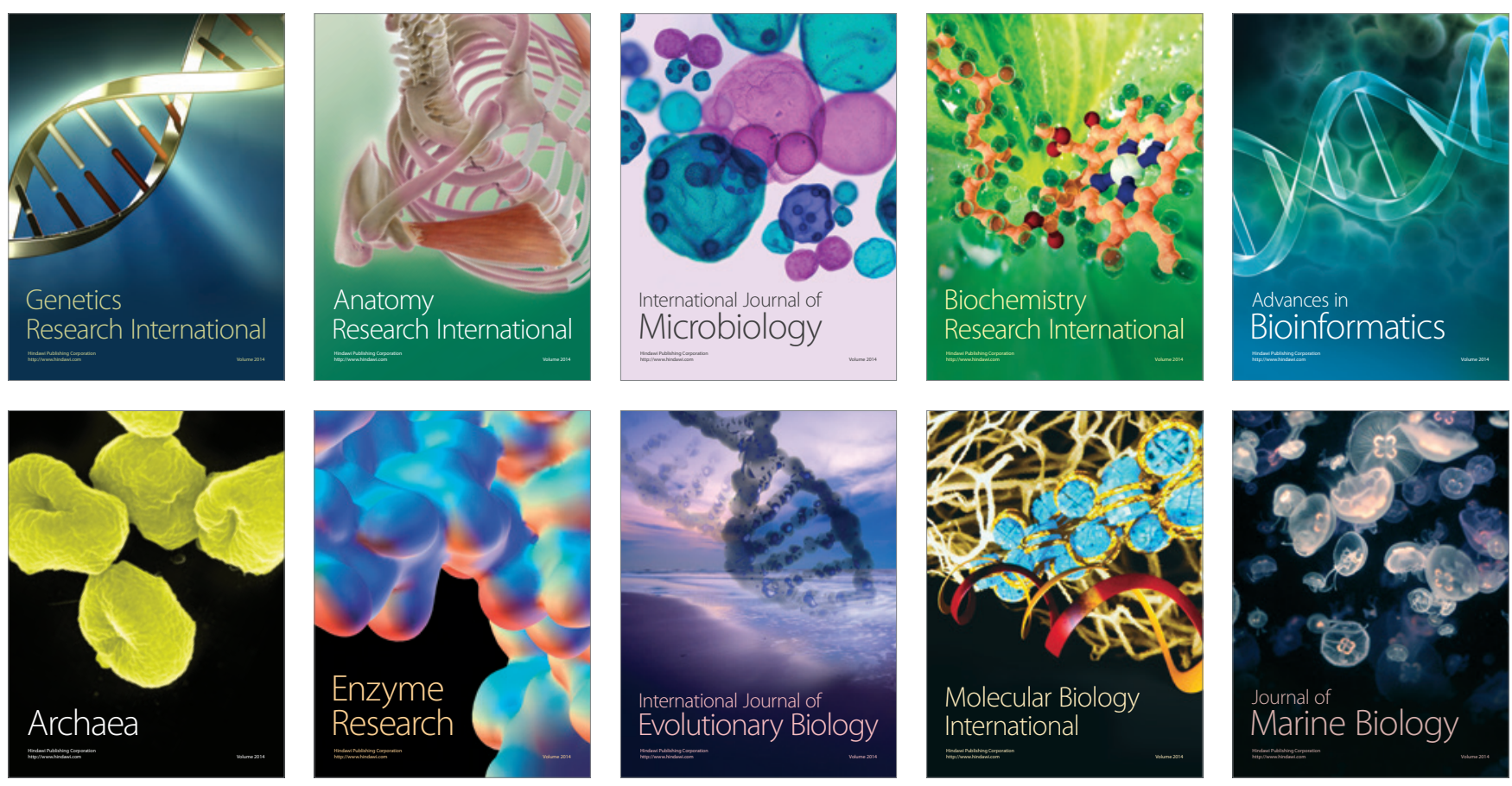